\title{
Variations on the Six Exponentials Theorem
}

\author{
Michel Waldschmidt
}

\begin{abstract}
According to the Four Exponentials Conjecture, a $2 \times$ 2 matrix whose entries $\lambda_{i j}(i=1,2, j=1,2)$ are logarithms of algebraic numbers is regular, as soon as the two rows as well as the two columns are linearly independent over the field $\mathbb{Q}$ of rational numbers. The question we address is as follows: are the numbers

$\lambda_{12}-\left(\lambda_{11} \lambda_{22} / \lambda_{21}\right), \quad\left(\lambda_{11} \lambda_{22}\right) /\left(\lambda_{12} \lambda_{21}\right), \quad\left(\lambda_{12} / \lambda_{11}\right)-\left(\lambda_{22} / \lambda_{21}\right)$

and

transcendental?$$
\lambda_{11} \lambda_{22}-\lambda_{21} \lambda_{12}
$$

Denote by $\widetilde{\mathcal{L}}$ the set of linear combination, with algebraic coefficients, of 1 and logarithms of algebraic numbers. A strong form of the Four Exponentials Conjecture states that a $2 \times 2$ matrix whose entries are in $\widetilde{\mathcal{L}}$ is regular, as soon as the two rows as well as the two columns are linearly independent over the field $\overline{\mathbb{Q}}$ of algebraic numbers. From this conjecture follows a positive answer (apart from trivial cases) to the previous question for the first three numbers: not only they are transcendental, but, even more, they are not in the set $\widetilde{\mathcal{L}}$. This Strong Four Exponentials Conjecture does not seem sufficient to settle the question for the last number, which amounts to prove that a $3 \times 3$ matrix is regular; the Conjecture of algebraic independence of logarithms of algebraic numbers provides the answer.
\end{abstract}

2000 Mathematics Subject Classification. 11J81 11J86 11J89.

Key words and phrases. Transcendental numbers, logarithms of algebraic numbers, four exponentials Conjecture, six exponentials Theorem, algebraic independence, elliptic functions, periods of $K 3$-surfaces.

The author is thankful to the organizing committee of the International Conference on Algebra and Number Theory, especially its secretary Rajat Tandon, for their invitation, to the faculty, staff and students of Hyderabad University for the excellent job they did to welcome the participants, to Stephane Fischler for his comments on a preliminary version of this paper, to Guy Diaz whose suggestions enabled the author to improve the results and to Hironori Shiga for contributing with his appendix. 
The first goal of this paper is to give the state of the art on these questions: we replace the Strong Four Exponentials Conjecture by the Strong Six Exponentials Theorem of D. Roy; we deduce that in a set of 2 numbers, at least one element is not in $\widetilde{\mathcal{L}}$ (and therefore is transcendental). The second goal is to replace the Conjecture of algebraic independence of logarithms by the Linear Subgroup Theorem; we obtain partial results on the non existence of quadratic relations among logarithms of algebraic numbers. The third and last goal is to consider elliptic analogs of these statements.

An appendix by Hironori Shiga provides a link with periods of $K 3$-surfaces.

\section{Conjectures - Exponential Case}

Denote by $\overline{\mathbb{Q}}$ the field of algebraic numbers (algebraic closure of $\mathbb{Q}$ in $\mathbb{C}$ ) and by $\mathcal{L}$ the $\mathbb{Q}$-vector space of logarithms of algebraic numbers:

$$
\mathcal{L}=\left\{\lambda \in \mathbb{C} ; e^{\lambda} \in \overline{\mathbb{Q}}^{\times}\right\}=\left\{\log \alpha ; \alpha \in \overline{\mathbb{Q}}^{\times}\right\}=\exp ^{-1}\left(\overline{\mathbb{Q}}^{\times}\right) .
$$

Here is the main Conjecture (see for instance [5], Historical Note of Chapter III, [4], Chap. 6 p. 259 and [12], Conjecture 1.15):

Conjecture 1.1. (Algebraic Independence of Logarithms of Algebraic Numbers). Let $\lambda_{1}, \ldots, \lambda_{n}$ be $\mathbb{Q}$-linearly independent elements of $\mathcal{L}$. Then $\lambda_{1}, \ldots, \lambda_{n}$ are algebraically independent.

The following special case of Conjecture 1.1 was already investigated by Th. Schneider ([9], end of Chap. 5), S. Lang ([5], Chap. II $\S 1)$ and K. Ramachandra ([6] II $\S 4$ ) in the 1960's (see [12], Conjecture 1.13):

ConjeCture 1.2. (Four Exponentials Conjecture). Let $M$ be a $2 \times 2$ matrix with entries in $\mathcal{L}$. Assume that the two rows of $M$ are linearly independent over $\mathbb{Q}$ and also that the two columns of $M$ are linearly independent over $\mathbb{Q}$. Then $M$ has rank 2 .

It is plain that a $2 \times 2$ matrix

$$
M=\left(\begin{array}{ll}
\lambda_{11} & \lambda_{12} \\
\lambda_{21} & \lambda_{22}
\end{array}\right)
$$

has rank $<2$ if and only if there exist $x_{1}, x_{2}, y_{1}, y_{2}$ such that $\lambda_{i j}=$ $x_{i} y_{j}(i=1,2, j=1,2)$. Hence Conjecture 1.2 is equivalent to the following statement: if $x_{1}, x_{2}$ are two complex numbers which are $\mathbb{Q}$ linearly independent and if $y_{1}, y_{2}$ are two complex numbers which are also $\mathbb{Q}$-linearly independent, then one at least of the four numbers

$$
e^{x_{1} y_{1}}, e^{x_{1} y_{2}}, e^{x_{2} y_{1}}, e^{x_{2} y_{2}}
$$


is transcendental.

Let $\lambda_{i j}(i=1,2, j=1,2)$ be four non-zero elements of $\mathcal{L}$. Assume that the two rows of the matrix (1.3) are linearly independent over $\mathbb{Q}$ and also that the two columns are linearly independent over $\mathbb{Q}$. The Four Exponentials Conjecture 1.2 states that each of the following four numbers is not zero:

$$
\lambda_{12}-\frac{\lambda_{11} \lambda_{22}}{\lambda_{21}}, \quad 1-\frac{\lambda_{11} \lambda_{22}}{\lambda_{21} \lambda_{12}}, \quad \frac{\lambda_{12}}{\lambda_{11}}-\frac{\lambda_{22}}{\lambda_{21}}, \quad \lambda_{11} \lambda_{22}-\lambda_{21} \lambda_{12}
$$

We investigate the transcendence of these numbers. From Conjecture 1.1, it follows that each of them is algebraic only in trivial cases (where in fact it is rational). More precisely, we show that the diophantine nature (rational, algebraic irrational or transcendental) of each of the three first numbers in (1.4) is settled by the following special case of Conjecture 1.1, suggested by D. Roy (see for instance [12] Conjecture 11.17).

Denote by $\widetilde{\mathcal{L}}$ the $\overline{\mathbb{Q}}$-vector space spanned by 1 and $\mathcal{L}$ in $\mathbb{C}$. Hence $\widetilde{\mathcal{L}}$ is the set of linear combinations of logarithms of algebraic numbers with algebraic coefficients:

$$
\begin{aligned}
\widetilde{\mathcal{L}}=\left\{\beta_{0}+\beta_{1} \log \alpha_{1}+\cdots+\beta_{n} \log \alpha_{n}\right. & \\
& \left.n \geq 0,\left(\alpha_{1}, \ldots, \alpha_{n}\right) \in\left(\overline{\mathbb{Q}}^{\times}\right)^{n},\left(\beta_{0}, \beta_{1}, \ldots, \beta_{n}\right) \in \overline{\mathbb{Q}}^{n+1}\right\} .
\end{aligned}
$$

Conjecture 1.5. (Strong Four Exponentials Conjecture). Let $M$ be a $2 \times 2$ matrix with entries in $\widetilde{\mathcal{L}}$. Assume that the two rows of $M$ are linearly independent over $\overline{\mathbb{Q}}$ and also that the two columns of $M$ are linearly independent over $\overline{\mathbb{Q}}$. Then $M$ has rank 2 .

Equivalently, if $x_{1}, x_{2}$ are two complex numbers which are $\overline{\mathbb{Q}}$-linearly independent and if $y_{1}, y_{2}$ are two complex numbers which are also $\overline{\mathbb{Q}}$ linearly independent, then one at least of the four numbers

$$
x_{1} y_{1}, x_{1} y_{2}, x_{2} y_{1}, x_{2} y_{2}
$$

does not belong to $\widetilde{\mathcal{L}}$.

We derive several consequences of the Strong Four Exponentials Conjecture 1.5 .

CONSEQUence 1.6. Let $\Lambda$ be a transcendental element of $\widetilde{\mathcal{L}}$. Then $1 / \Lambda$ is not in $\widetilde{\mathcal{L}}$.

COnsequence 1.7. Let $\Lambda_{1}, \Lambda_{2}$ be two elements of $\widetilde{\mathcal{L}}$. Assume that $\Lambda_{1}$ and $\Lambda_{2} / \Lambda_{1}$ are transcendental. Then this quotient $\Lambda_{2} / \Lambda_{1}$ is not in $\widetilde{\mathcal{L}}$. 
CONSEQUENCE 1.8. Let $\Lambda_{1}, \Lambda_{2}$ be two transcendental elements of $\widetilde{\mathcal{L}}$. Then the product $\Lambda_{1} \Lambda_{2}$ is not in $\widetilde{\mathcal{L}}$.

Consequence 1.9. Let $\Lambda_{1}, \Lambda_{2}, \Lambda_{3}$ be three elements of $\widetilde{\mathcal{L}}$ with $\Lambda_{2} \neq$ 0 . Assume that the two numbers $\Lambda_{1} / \Lambda_{2}$ and $\Lambda_{3} / \Lambda_{2}$ are transcendental. Then

$$
\frac{\Lambda_{1} \Lambda_{3}}{\Lambda_{2}} \notin \widetilde{\mathcal{L}}
$$

Examples where Consequences 1.7, 1.8 and 1.9 hold unconditionally (i.e. without assuming Conjecture 1.5) are given in $\S 2$ below (see Corollaries 2.7, 2.8 and 2.9).

Proof of Consequences 1.6, 1.7, 1.8 And 1.9 Assuming Conjecture 1.5. Let $\Lambda_{0}$ be an element in $\widetilde{\mathcal{L}}$. We apply the Strong Four Exponentials Conjecture 1.5 to the matrices

$$
\left(\begin{array}{cc}
\Lambda & 1 \\
1 & \Lambda_{0}
\end{array}\right), \quad\left(\begin{array}{cc}
\Lambda_{1} & \Lambda_{2} \\
1 & \Lambda_{0}
\end{array}\right), \quad\left(\begin{array}{cc}
1 & \Lambda_{2} \\
\Lambda_{1} & \Lambda_{0}
\end{array}\right) \quad \text { and } \quad\left(\begin{array}{cc}
\Lambda_{2} & \Lambda_{3} \\
\Lambda_{1} & \Lambda_{0}
\end{array}\right)
$$

Since $1 \in \widetilde{\mathcal{L}}$ one may also deduce Consequences 1.7 and 1.8 from 1.9 and then 1.6 from 1.7 .

We recall Baker's Theorem on linear independence of logarithms of algebraic numbers: if $\lambda_{1}, \ldots, \lambda_{n}$ are $\mathbb{Q}$-linearly independent elements of $\mathcal{L}$, then the numbers $1, \lambda_{1}, \ldots, \lambda_{n}$ are $\overline{\mathbb{Q}}$-linearly independent.

Consequence 1.9 applies to the three first numbers in (1.4). Let $\lambda_{i j}$ $(i=1,2, j=1,2)$ be four non-zero elements of $\mathcal{L}$. Assuming Conjecture 1.5 , we deduce

$$
\lambda_{12}-\frac{\lambda_{11} \lambda_{22}}{\lambda_{21}} \notin \overline{\mathbb{Q}} \backslash\{0\}, \quad \frac{\lambda_{11} \lambda_{22}}{\lambda_{12} \lambda_{21}} \notin \overline{\mathbb{Q}} \backslash \mathbb{Q}
$$

and

$$
\frac{\lambda_{12}}{\lambda_{11}}-\frac{\lambda_{22}}{\lambda_{21}} \notin \overline{\mathbb{Q}} \backslash \mathbb{Q} \text {. }
$$

Moreover, again under Conjecture 1.5, if the number

is rational, then

$$
\frac{\lambda_{12}}{\lambda_{11}}-\frac{\lambda_{22}}{\lambda_{21}}
$$

- either $\lambda_{12} / \lambda_{11} \in \mathbb{Q}$ and $\lambda_{22} / \lambda_{21} \in \mathbb{Q}$

- or $\lambda_{21} / \lambda_{11} \in \mathbb{Q}$.

Notice that if $\lambda_{11}$ and $\lambda_{12}$ are two elements of $\mathcal{L}$ and $a, b$ two rational numbers with $b \lambda_{11} \neq 0$, then

$$
\frac{\lambda_{12}}{\lambda_{11}}-\frac{b \lambda_{12}-a \lambda_{11}}{b \lambda_{11}}=\frac{a}{b} .
$$


Consequence 1.8 shows that, if Conjecture 1.5 holds, and if $\lambda_{11}, \lambda_{12}, \lambda_{22}$ are three elements of $\mathcal{L}$ such that $\lambda_{11} \lambda_{22} \neq 0$, then both numbers

$$
\lambda_{11} \lambda_{22}-\lambda_{12} \text { and } \frac{\lambda_{12}}{\lambda_{11}}-\lambda_{22}
$$

are transcendental.

The transcendence of the last number $\lambda_{11} \lambda_{22}-\lambda_{21} \lambda_{12}$ in (1.4) does not seem to follow from the Strong Four Exponentials Conjecture. However Conjecture 1.1 claims that any algebraic relation among logarithms of algebraic numbers is homogeneous, hence:

Consequence 1.10. (of Conjecture 1.1). Let $\lambda_{i j}(i=1,2, j=1,2)$ be four transcendental elements of $\mathcal{L}$. Assume

$$
\lambda_{11} \lambda_{22} \neq \lambda_{21} \lambda_{12}
$$

Then the number

$$
\lambda_{11} \lambda_{22}-\lambda_{21} \lambda_{12}
$$

is not in $\widetilde{\mathcal{L}}$.

Consequence 1.10 amounts to study certain quadratic relations among logarithms of algebraic numbers. One may deduce it directly from the next conjecture of D. Roy on the rank of matrices whose entries are in $\widetilde{\mathcal{L}}$ (see [8], remarks (i) and (ii) p. 54, as well as section 12.1.4 in [12]).

Let $M$ be a $d \times \ell$ matrix with entries in $\widetilde{\mathcal{L}}$. Let $\Lambda_{1}, \ldots, \Lambda_{s}$ be a basis over $\overline{\mathbb{Q}}$ of the vector space spanned by these entries. Write

$$
M=M_{1} \Lambda_{1}+\cdots+M_{s} \Lambda_{s},
$$

where $M_{1}, \ldots, M_{s}$ are $d \times \ell$ matrices with algebraic entries. The structural rank of $M$ with respect to $\overline{\mathbb{Q}}$ is defined as the rank of the matrix

$$
M_{1} X_{1}+\cdots+M_{s} X_{s}
$$

whose entries are in the field $\overline{\mathbb{Q}}\left(X_{1}, \ldots, X_{s}\right)$. This definition is independent of the chosen basis $\Lambda_{1}, \ldots, \Lambda_{s}$.

As noted by D. Roy (see [12] Prop. 12.13), Conjecture 1.1 is equivalent to the next statement:

CONJECTURE 1.11. The rank of a matrix with entries in $\widetilde{\mathcal{L}}$ is equal to its structural rank with respect to $\overline{\mathbb{Q}}$.

By homogeneity, for any $\lambda_{i j}(i=1,2, j=1,2)$ in $\mathcal{L} \backslash\{0\}$ with

$$
\lambda_{11} \lambda_{22} \neq \lambda_{12} \lambda_{21}
$$


and for any $\Lambda \in \widetilde{\mathcal{L}} \backslash\{0\}$ the structural rank with respect to $\overline{\mathbb{Q}}$ of

$$
\left(\begin{array}{ccc}
1 & 0 & \lambda_{11} \\
0 & 1 & \lambda_{12} \\
\lambda_{22} & -\lambda_{21} & \Lambda
\end{array}\right)
$$

is 3. Hence Conjecture 1.11 implies Consequence 1.10.

The two next statements, which are consequences of Conjecture 1.11, involve cubic relations among logarithms of algebraic numbers.

Consequence 1.12. Let $\lambda_{i j}(i=1,2, j=1,2)$ be four non-zero elements of $\mathcal{L}$. Then

$$
\frac{\lambda_{12}}{\lambda_{11}}-\frac{\lambda_{22}}{\lambda_{21}}
$$

is not in $\widetilde{\mathcal{L}} \backslash \mathbb{Q}$.

Consequence 1.13. Let $\lambda_{i j}(i=1,2, j=1,2)$ be four non-zero elements of $\mathcal{L}$. Then

$$
\frac{\lambda_{11} \lambda_{22}}{\lambda_{21} \lambda_{12}}
$$

is not in $\widetilde{\mathcal{L}} \backslash \mathbb{Q}$.

One deduces Consequences 1.12 and 1.13 from Conjecture 1.11 by introducing the matrices

$$
\left(\begin{array}{ccc}
\lambda_{11} & \lambda_{12} & 0 \\
\lambda_{21} & \lambda_{22} & \Lambda \\
0 & \lambda_{21} & 1
\end{array}\right) \text { and }\left(\begin{array}{ccc}
\lambda_{11} & \lambda_{12} & 0 \\
\lambda_{21} & 0 & 1 \\
0 & \lambda_{22} & \Lambda
\end{array}\right)
$$

with $\Lambda \in \widetilde{\mathcal{L}}$.

\section{Theorems - Exponential Case}

The sharpest known result in direction of the Strong Four Exponentials Conjecture 1.5 is the following one, due to D. Roy ([7] Corollary 2 $\S 4$ p. 38; see also [12] Corollary 11.16).

Theorem 2.1. (Strong Six Exponentials Theorem). Let $M$ be a $2 \times 3$ matrix with entries in $\widetilde{\mathcal{L}}$. Assume that the two rows of $M$ are linearly independent over $\overline{\mathbb{Q}}$ and also that the three columns of $M$ are linearly independent over $\overline{\mathbb{Q}}$. Then $M$ has rank 2 .

Equivalently, if $x_{1}, x_{2}$ are two complex numbers which are $\overline{\mathbb{Q}}$-linearly independent and if $y_{1}, y_{2}, y_{3}$ are three complex numbers which are also $\overline{\mathbb{Q}}$-linearly independent, then one at least of the six numbers

$$
x_{1} y_{1}, x_{1} y_{2}, x_{1} y_{3}, x_{2} y_{1}, x_{2} y_{2}, x_{2} y_{3}
$$


does not belong to $\widetilde{\mathcal{L}}$.

We select a sample of consequences of Theorem 2.1 related to the conjectural statements of $\S 1$.

The first one involves the inverse of an element in $\widetilde{\mathcal{L}}$, like in Consequence 1.6, and also a quotient of two elements in $\widetilde{\mathcal{L}}$, like in Consequence 1.7 .

Corollary 2.2. Let $\Lambda_{1}, \Lambda_{2}$ be two elements of $\widetilde{\mathcal{L}}$. Assume that the three numbers $1, \Lambda_{1}, \Lambda_{2}$ are $\overline{\mathbb{Q}}$-linearly independent. Then one at least of the two numbers $1 / \Lambda_{1}, \Lambda_{2} / \Lambda_{1}$ is not in $\widetilde{\mathcal{L}}$.

The second corollary deals with two quotients of elements in $\widetilde{\mathcal{L}}$ and provides a partial answer to Consequence 1.7.

Corollary 2.3. Let $\Lambda_{1}, \Lambda_{2}, \Lambda_{3}$ be three elements of $\widetilde{\mathcal{L}}$ Assume that $\Lambda_{1}$ is transcendental and that the three numbers $\Lambda_{1}, \Lambda_{2}, \Lambda_{3}$ are $\overline{\mathbb{Q}}$-linearly independent. Then one at least of the two numbers $\Lambda_{2} / \Lambda_{1}$, $\Lambda_{3} / \Lambda_{1}$ is not in $\widetilde{\mathcal{L}}$.

The third one involves two products of elements in $\widetilde{\mathcal{L}}$ and therefore is related to Consequence 1.8.

Corollary 2.4. Let $\Lambda_{1}, \Lambda_{2}, \Lambda_{3}$ be three transcendental elements of $\widetilde{\mathcal{L}}$ Assume that the three numbers $1, \Lambda_{2}, \Lambda_{3}$ are $\overline{\mathbb{Q}}$-linearly independent. Then one at least of the two numbers $\Lambda_{1} \Lambda_{2}, \Lambda_{1} \Lambda_{3}$ is not in $\widetilde{\mathcal{L}}$.

The next corollary combines Consequence 1.7 and Consequence 1.9.

COROLlary 2.5. Let $\Lambda_{1}, \Lambda_{2}, \Lambda_{3}$ be three transcendental elements of $\widetilde{\mathcal{L}}$ Assume that $\Lambda_{1} / \Lambda_{2}$ is transcendental and that the three numbers 1 , $\Lambda_{2}, \Lambda_{3}$ are $\overline{\mathbb{Q}}$-linearly independent. Then one at least of the two numbers $\Lambda_{1} / \Lambda_{2}, \Lambda_{1} \Lambda_{3} / \Lambda_{2}$ is not in $\widetilde{\mathcal{L}}$.

The last one is a weak but unconditional version of Consequence 1.9.

Corollary 2.6. Let $\Lambda_{1}, \Lambda_{2}, \Lambda_{3}, \Lambda_{4}$ be four elements of $\widetilde{\mathcal{L}}$. Assume that $\Lambda_{1} / \Lambda_{2}$ is transcendental and that the three numbers $\Lambda_{2}, \Lambda_{3}, \Lambda_{4}$ are linearly independent over $\overline{\mathbb{Q}}$. Then one at least of the two numbers

$$
\frac{\Lambda_{1} \Lambda_{3}}{\Lambda_{2}}, \frac{\Lambda_{1} \Lambda_{4}}{\Lambda_{2}}
$$

is not in $\widetilde{\mathcal{L}}$.

Proof of Corollaries 2.2, 2.3, 2.4, 2.5 And 2.6. We apply Theorem 2.1 to the matrices

$$
\left(\begin{array}{ccc}
\Lambda_{1} & 1 & \Lambda_{2} \\
1 & \Lambda & \Lambda^{\prime}
\end{array}\right), \quad\left(\begin{array}{ccc}
\Lambda_{1} & \Lambda_{2} & \Lambda_{3} \\
1 & \Lambda & \Lambda^{\prime}
\end{array}\right), \quad\left(\begin{array}{ccc}
1 & \Lambda_{2} & \Lambda_{3} \\
\Lambda_{1} & \Lambda & \Lambda^{\prime}
\end{array}\right),
$$




$$
\left(\begin{array}{ccc}
\Lambda_{2} & \Lambda_{3} & 1 \\
\Lambda_{1} & \Lambda & \Lambda^{\prime}
\end{array}\right), \quad\left(\begin{array}{ccc}
\Lambda_{2} & \Lambda_{3} & \Lambda_{4} \\
\Lambda_{1} & \Lambda & \Lambda^{\prime}
\end{array}\right)
$$

with $\Lambda$ and $\Lambda^{\prime}$ in $\widetilde{\mathcal{L}}$.

From Corollary 2.4 we deduce an example due to G. Diaz [3] where the statement 1.8 holds. See also [2]. The basic idea occurs initially in [6] I p. 68: a complex number is algebraic if and only if both its real part and its imaginary part are algebraic.

Corollary 2.7. (G. Diaz). Let $\Lambda_{1} \in \widetilde{\mathcal{L}} \cap(\mathbb{R} \cup i \mathbb{R}) \backslash \overline{\mathbb{Q}}$ and let $\Lambda_{2} \in \widetilde{\mathcal{L}}$. Assume that the three numbers $1, \Lambda_{2}$ and $\bar{\Lambda}_{2}$ are linearly independent over $\overline{\mathbb{Q}}$. Then the product $\Lambda_{1} \Lambda_{2}$ is not in $\widetilde{\mathcal{L}}$.

Indeed Corollary 2.4 with $\Lambda_{3}=\bar{\Lambda}_{2}$ shows that one at least of the two numbers $\Lambda_{1} \Lambda_{2}, \Lambda_{1} \bar{\Lambda}_{2}$ is not in $\widetilde{\mathcal{L}}$. From the assumption $\Lambda_{1}= \pm \bar{\Lambda}_{1}$ the result follows.

Using Hermite-Lindemann's Theorem

$$
\mathcal{L} \cap \overline{\mathbb{Q}}=\{0\},
$$

we deduce that for $\Lambda_{1} \in \widetilde{\mathcal{L}} \cap(\mathbb{R} \cup i \mathbb{R}) \backslash \overline{\mathbb{Q}}$ and $\lambda_{2} \in \mathcal{L} \backslash(\mathbb{R} \cup i \mathbb{R})$, the product $\Lambda_{1} \lambda_{2}$ is not in $\widetilde{\mathcal{L}}$.

In the same vein we may produce examples where Consequences 1.7 and 1.9 hold. We give only two such examples.

Corollary 2.8. Let $\Lambda_{1} \in \widetilde{\mathcal{L}} \cap(\mathbb{R} \cup i \mathbb{R}) \backslash \overline{\mathbb{Q}}$ and $\Lambda_{2} \in \widetilde{\mathcal{L}}$. Assume that the three numbers $\Lambda_{1}, \Lambda_{2}$ and $\bar{\Lambda}_{2}$ are linearly independent over $\overline{\mathbb{Q}}$. Then the quotient $\Lambda_{2} / \Lambda_{1}$ is not in $\widetilde{\mathcal{L}}$.

COROLlary 2.9. Let $\Lambda_{1}, \Lambda_{2}, \Lambda_{3}$ be three elements in $\widetilde{\mathcal{L}}$ with $\Lambda_{1}$ and $\Lambda_{2}$ in $\mathbb{R} \cup i \mathbb{R}$ and $\Lambda_{2} \neq 0$. Assume that $\Lambda_{1} / \Lambda_{2}$ is transcendental and that the three numbers $\Lambda_{2}, \Lambda_{3}$ and $\bar{\Lambda}_{3}$ are $\overline{\mathbb{Q}}$-linearly independent. Then $\Lambda_{1} \Lambda_{3} / \Lambda_{2}$ is not in $\widetilde{\mathcal{L}}$.

One deduces from Corollary 2.6 the following results. Let $M$ be a $2 \times 3$ matrix with entries in $\mathcal{L}$ :

$$
M=\left(\begin{array}{lll}
\lambda_{11} & \lambda_{12} & \lambda_{13} \\
\lambda_{21} & \lambda_{22} & \lambda_{23}
\end{array}\right)
$$

Assume that the two numbers $\lambda_{11}, \lambda_{21}$ are $\mathbb{Q}$-linearly independent, and also that the three numbers $\lambda_{21}, \lambda_{22}, \lambda_{23}$ are $\mathbb{Q}$-linearly independent. Then

(i) one at least of the two numbers

$$
\lambda_{12}-\frac{\lambda_{11} \lambda_{22}}{\lambda_{21}}, \quad \lambda_{13}-\frac{\lambda_{11} \lambda_{23}}{\lambda_{21}}
$$


is transcendental,

(ii) if $\lambda_{12} \lambda_{13} \neq 0$, then one at least of the two numbers

$$
\frac{\lambda_{11} \lambda_{22}}{\lambda_{21} \lambda_{12}}, \frac{\lambda_{11} \lambda_{23}}{\lambda_{21} \lambda_{13}}
$$

is transcendental,

(iii) one at least of the two numbers

$$
\frac{\lambda_{12}}{\lambda_{11}}-\frac{\lambda_{22}}{\lambda_{21}}, \frac{\lambda_{13}}{\lambda_{11}}-\frac{\lambda_{23}}{\lambda_{21}}
$$

is transcendental

and

(iv) one at least of the two numbers

$$
\frac{\lambda_{11}}{\lambda_{21}}-\frac{\lambda_{12}}{\lambda_{22}}, \quad \frac{\lambda_{11}}{\lambda_{21}}-\frac{\lambda_{13}}{\lambda_{23}}
$$

is transcendental.

From Corollary 2.4 it follows that, if $\lambda_{11}, \lambda_{12}, \lambda_{13}$ are three elements of $\mathcal{L}$ with $\lambda_{11} \neq 0$ and if the two numbers $\lambda_{22}, \lambda_{23}$ are $\mathbb{Q}$-linearly independent elements of $\mathcal{L}$, then

(i) one at least of the two numbers

$$
\lambda_{11} \lambda_{22}-\lambda_{12}, \quad \lambda_{11} \lambda_{23}-\lambda_{13}
$$

is transcendental

and

(ii) one at least of the two numbers

$$
\frac{\lambda_{12}}{\lambda_{11}}-\lambda_{22}, \quad \frac{\lambda_{13}}{\lambda_{11}}-\lambda_{23}
$$

is transcendental.

For a $2 \times 3$ matrix $(2.10)$ with entries in $\mathcal{L}$, assuming that on each row and on each column the entries are linearly independent over $\mathbb{Q}$, one would like to prove that one at least of the two numbers

$$
\lambda_{11} \lambda_{22}-\lambda_{21} \lambda_{12}, \quad \lambda_{11} \lambda_{23}-\lambda_{21} \lambda_{13}
$$

is transcendental (see Corollary 2.13 for a special case). In a forthcoming paper we shall prove that one at least of the three $2 \times 2$ determinants

$$
\lambda_{11} \lambda_{22}-\lambda_{21} \lambda_{12}, \quad \lambda_{11} \lambda_{23}-\lambda_{21} \lambda_{13}, \quad \lambda_{12} \lambda_{23}-\lambda_{22} \lambda_{13}
$$

is not in $\widetilde{\mathcal{L}}$.

A partial answer will follow from the next result, whose proof is given in $\S 3$. 
Theorem 2.11. Let $M=\left(\Lambda_{i j}\right)_{1 \leq i \leq m ; 1 \leq j \leq \ell}$ be a $m \times \ell$ matrix with entries in $\widetilde{\mathcal{L}}$. Denote by $I_{m}$ the identity $m \times m$ matrix and assume that the $m+\ell$ column vectors of the matrix $\left(I_{m}, M\right)$ are linearly independent over $\overline{\mathbb{Q}}$. Let $\Lambda_{1}, \ldots, \Lambda_{m}$ be elements of $\widetilde{\mathcal{L}}$. Assume that the numbers 1 , $\Lambda_{1}, \ldots, \Lambda_{m}$ are $\overline{\mathbb{Q}}$-linearly independent. Assume further $\ell>m^{2}$. Then one at least of the $\ell$ numbers

$$
\Lambda_{1} \Lambda_{1 j}+\cdots+\Lambda_{m} \Lambda_{m j} \quad(j=1, \ldots, \ell)
$$

is not in $\widetilde{\mathcal{L}}$.

Taking $m=1, \ell=2$ in Theorem 2.11 we recover Corollary 2.4. In case $m=2, \ell=5$ we deduce the next two corollaries.

Corollary 2.12. Let $M$ be a $2 \times 5$ matrix with entries in $\widetilde{\mathcal{L}}$ :

$$
\left(\begin{array}{lllll}
\Lambda_{11} & \Lambda_{12} & \Lambda_{13} & \Lambda_{14} & \Lambda_{15} \\
\Lambda_{21} & \Lambda_{22} & \Lambda_{23} & \Lambda_{24} & \Lambda_{25}
\end{array}\right)
$$

Assume that $\Lambda_{21} \neq 0$, that the number $\Lambda_{11} / \Lambda_{21}$ is transcendental and that the seven columns of the matrix $\left(I_{2}, M\right)$ are linearly independent over $\overline{\mathbb{Q}}$. Then one at least of the four numbers

$$
\Lambda_{11} \Lambda_{2 j}-\Lambda_{21} \Lambda_{1 j} \quad(j=2,3,4,5)
$$

is not in $\widetilde{\mathcal{L}}$.

Proof. In Theorem 2.11 we set

$$
m=2, \quad \ell=5, \quad \Lambda_{1}=-\Lambda_{21} \quad \text { and } \quad \Lambda_{2}=\Lambda_{11} .
$$

By adding further hypotheses and by using the complex conjugation à la Diaz we can reduce from 4 to 2 the number of elements in the set which we show not to be included in $\widetilde{\mathcal{L}}$ :

Corollary 2.13. Let $M$ be a $2 \times 3$ matrix with entries in $\widetilde{\mathcal{L}}$ :

$$
\left(\begin{array}{lll}
\Lambda_{11} & \Lambda_{12} & \Lambda_{13} \\
\Lambda_{21} & \Lambda_{22} & \Lambda_{23}
\end{array}\right)
$$

Assume that $\Lambda_{11}, \Lambda_{12}, \Lambda_{13}$ and $\Lambda_{21}$ are in $\mathbb{R} \cup i \mathbb{R}$ and that $\Lambda_{21} \neq 0$. Assume further that the number $\Lambda_{11} / \Lambda_{21}$ is transcendental and furthermore that the six numbers $1, \Lambda_{21}, \Lambda_{22}, \Lambda_{23}, \bar{\Lambda}_{22}, \bar{\Lambda}_{23}$ are linearly independent over $\overline{\mathbb{Q}}$. Then one at least of the two numbers

$$
\Lambda_{11} \Lambda_{22}-\Lambda_{21} \Lambda_{12}, \quad \Lambda_{11} \Lambda_{23}-\Lambda_{21} \Lambda_{13}
$$

is not in $\widetilde{\mathcal{L}}$. 
Proof. For $j=2$ and $j=3$ define

$$
\Lambda_{j}=\Lambda_{11} \Lambda_{2 j}-\Lambda_{21} \Lambda_{1 j}
$$

We apply Corollary 2.12 to the $2 \times 5$ matrix

$$
\left(\begin{array}{ccccc}
\Lambda_{11} & \Lambda_{12} & \Lambda_{13} & \bar{\Lambda}_{12} & \bar{\Lambda}_{13} \\
\Lambda_{21} & \Lambda_{22} & \Lambda_{23} & \bar{\Lambda}_{22} & \bar{\Lambda}_{23}
\end{array}\right)
$$

with

$$
\Lambda_{14}=\bar{\Lambda}_{12}, \quad \Lambda_{15}=\bar{\Lambda}_{13}, \quad \Lambda_{24}=\bar{\Lambda}_{22}, \quad \Lambda_{25}=\bar{\Lambda}_{23}
$$

We deduce that one at least of the four numbers $\Lambda_{2}, \bar{\Lambda}_{2}, \Lambda_{3}, \bar{\Lambda}_{3}$ is not in $\widetilde{\mathcal{L}}$, hence one at least of the two numbers $\Lambda_{2}, \Lambda_{3}$ is not in $\widetilde{\mathcal{L}}$.

We conclude this section with the following consequence of Theorem 2.11, whose conclusion goes further than [12] Exercise 11.10 (which is [11] Corollary 2.5).

Corollary 2.14. Let $M=\left(\lambda_{i j}\right)_{1 \leq i \leq m ; 1 \leq j \leq \ell}$ be a $m \times \ell$ matrix with entries in $\mathcal{L}$ whose columns are linearly independent over $\mathbb{Q}$ and let $\lambda_{1}, \ldots, \lambda_{m}$ be $\mathbb{Q}$-linearly independent elements in $\mathcal{L}$. Assume $\ell>m^{2}$. Then one at least of the $\ell$ numbers

$$
\lambda_{1} \lambda_{1 j}+\cdots+\lambda_{m} \lambda_{m j} \quad(j=1, \ldots, \ell)
$$

is not in $\widetilde{\mathcal{L}}$.

Proof. According to part (iii) of Exercise 11.5 in [12], Baker's homogeneous Theorem implies that $\mathbb{Q}$-linearly independent elements in $\mathcal{L}^{m}$ are linearly independent over $\overline{\mathbb{Q}}$. In the same way, using the full force of Baker's Theorem, we deduce that the columns of the matrix $\left(I_{m}, M\right)$ are linearly independent over $\overline{\mathbb{Q}}$. We use again Baker's Theorem to deduce from the assumptions of Corollary 2.14 that the numbers $1, \lambda_{1}, \ldots, \lambda_{m}$ are $\overline{\mathbb{Q}}$-linearly independent. Hence the hypotheses of Theorem 2.11 are fulfilled for $\Lambda_{i}=\lambda_{i}$ and $\Lambda_{i j}=\lambda_{i j}$.

\section{Proof of Theorem 2.11 and further results}

Theorem 5 of [7] (see also Corollary 11.15 in [12]) deals with the intersection $\mathcal{V} \cap \widetilde{\mathcal{L}}^{d}$ when $\mathcal{V}$ is a vector subspace of $\mathbb{C}^{d}$. For the proof of Theorem 2.11 we shall need only the special case where $\mathcal{V}$ is a hyperplane of $\mathbb{C}^{d}$.

TheOREM 3.1. Let $\mathcal{V}$ be a hyperplane in $\mathbb{C}^{d}$ such that $\mathcal{V} \cap \overline{\mathbb{Q}}^{d}=\{0\}$. Then $\mathcal{V} \cap \widetilde{\mathcal{L}}^{d}$ is a finite dimensional vector space over $\overline{\mathbb{Q}}$ of dimension $\leq d(d-1)$. 
Proof of Theorem 2.11. Let $d=m+1$. The hyperplane $\mathcal{V}$ of $\mathbb{C}^{d}$ of equation

$$
\Lambda_{1} z_{1}+\cdots+\Lambda_{m} z_{m}=z_{m+1}
$$

contains the $m+\ell$ following points:

$$
\gamma_{i}=\left(\epsilon_{i}, \Lambda_{i}\right) \quad(1 \leq i \leq m)
$$

and

$$
\gamma_{m+j}=\left(\Lambda_{1 j}, \ldots, \Lambda_{m j}, \Lambda_{j}^{\prime}\right) \quad(1 \leq j \leq \ell),
$$

where $\left(\epsilon_{1}, \ldots, \epsilon_{m}\right)$ is the canonical basis of $\mathbb{C}^{m}$ and where

$$
\Lambda_{j}^{\prime}=\Lambda_{1} \Lambda_{1 j}+\cdots+\Lambda_{m} \Lambda_{m j} \quad(j=1, \ldots, \ell) .
$$

The definition of $\Lambda_{1}^{\prime}, \ldots, \Lambda_{\ell}^{\prime}$ means that the $(m+1) \times(m+\ell)$ matrix

$$
\widetilde{M}=\left(\begin{array}{cccccc} 
& I_{m} & & & M & \\
\Lambda_{1} & \cdots & \Lambda_{m} & \Lambda_{1}^{\prime} & \cdots & \Lambda_{\ell}^{\prime}
\end{array}\right) .
$$

has rank $m$.

Since $1, \Lambda_{1}, \ldots, \Lambda_{m}$ are $\overline{\mathbb{Q}}$-linearly independent, we have $\mathcal{V} \cap \overline{\mathbb{Q}}^{d}=$ $\{0\}$. Since the columns of $\widetilde{M}$ are $\overline{\mathbb{Q}}$-linearly independent, the $m+\ell$ points $\gamma_{1}, \ldots, \gamma_{m+\ell}$ are linearly independent over $\overline{\mathbb{Q}}$. The assumption $\ell>m^{2}$ yields $m+\ell>d(d-1)$, hence Theorem 3.1 shows that one at least of the $\ell$ numbers $\Lambda_{1}^{\prime}, \ldots, \Lambda_{\ell}^{\prime}$ is not in $\widetilde{\mathcal{L}}$.

Another corollary of Theorem 3.1 is the following.

Corollary 3.2. Let $M=\left(\Lambda_{i j}\right)_{1 \leq i \leq m ; 1 \leq j \leq \ell}$ be a $m \times \ell$ matrix with entries in $\widetilde{\mathcal{L}}$ whose columns are linearly independent over $\overline{\mathbb{Q}}$ and let $x_{1}, \ldots, x_{m}$ be $\overline{\mathbb{Q}}$-linearly independent complex numbers. Assume $\ell>$ $m(m-1)$. Then one at least of the $\ell$ numbers

$$
x_{1} \Lambda_{1 j}+\cdots+x_{m} \Lambda_{m j} \quad(j=1, \ldots, \ell)
$$

is not zero.

Proof. Apply Theorem 3.1 with $d=m$ to the hyperplane $\mathcal{V}$ of equation

$$
x_{1} z_{1}+\cdots+x_{m} z_{m}=0
$$

in $\mathbb{C}^{m}$.

Further related results, which deserve to be compared with Theorem 2.11, follow from Corollary 11.6 in $[\mathbf{1 2}]$ which reads as follows. 
TheOREm 3.3. Let $d_{0} \geq 0$ and $d_{1} \geq 1$ be two positive integers. Set $d=d_{0}+d_{1}$. Le $\mathcal{V}$ be a vector subspace of $\mathbb{C}^{d}$ of dimension $n$ which satisfies

$$
\mathcal{V} \cap\left(\overline{\mathbb{Q}}^{d_{0}} \times\{0\}\right)=\{0\} \quad \text { and } \mathcal{V} \cap\left(\{0\} \times \mathbb{Q}^{d_{1}}\right)=\{0\} .
$$

Denote by $\ell_{0}$ the dimension of the $\overline{\mathbb{Q}}$-vector space $\mathcal{V} \cap \overline{\mathbb{Q}}^{d}$. Then the $\mathbb{Q}$-vector space $\mathcal{V} \cap\left(\overline{\mathbb{Q}}^{d_{0}} \times \mathcal{L}^{d_{1}}\right)$ has finite dimension bounded by

$$
\operatorname{dim}_{\mathbb{Q}}\left(\mathcal{V} \cap\left(\overline{\mathbb{Q}}^{d_{0}} \times \mathcal{L}^{d_{1}}\right)\right) \leq d_{1}\left(n-\ell_{0}\right) .
$$

A consequence of Theorem 3.3 is Corollary 1.6 in [11]. However there are two misprints in this last statement which we take the opportunity to correct here: firstly the assumption in Corollary 1.6 of [11] that $t_{1}, \ldots, t_{m}$ are $\mathbb{Q}$-linearly independent should be replaced by the assumption that $1, t_{1}, \ldots, t_{m}$ are $\mathbb{Q}$-linearly independent, and secondly in the condition $\ell>(r-1)(n+1)$ one should read $m$ in place of $n$. Here is an equivalent formulation of the corrected statement.

Corollary 3.4. Let $M=\left(\lambda_{i j}\right)_{1 \leq i \leq m ; 1 \leq j \leq \ell}$ be a $m \times \ell$ matrix with entries in $\mathcal{L}$ whose columns are linearly independent over $\mathbb{Q}$ and let $x_{1}, \ldots, x_{m}$ be $\mathbb{Q}$-linearly independent complex numbers. Denote by $r$ the dimension of the $\overline{\mathbb{Q}}$-vector space spanned by $x_{1}, \ldots, x_{m}$ and assume $\ell>m(r-1)$. Then one at least of the $\ell$ numbers

$$
x_{1} \lambda_{1 j}+\cdots+x_{m} \lambda_{m j} \quad(j=1, \ldots, \ell)
$$

is not zero.

If we use only the upper bound $r \leq m$ in Corollary 3.4, the result we obtain is a consequence of Corollary 3.2 (compare with part a) of Exercise 11.9 in $[\mathbf{1 2}]$ ).

Here is another consequence of Theorem 3.3 (with $d_{0}=1, d_{1}=n=$ $\left.m, \ell_{0}=m-r\right)$.

Corollary 3.5. Let $M=\left(\lambda_{i j}\right)_{1 \leq i \leq m ; 1 \leq j \leq \ell}$ be a $m \times \ell$ matrix with entries in $\mathcal{L}$ whose columns are linearly independent over $\mathbb{Q}$ and let $x_{1}, \ldots, x_{m}$ be $\mathbb{Q}$-linearly independent complex numbers. Denote by $r+1$ the dimension of the $\overline{\mathbb{Q}}$-vector space spanned by $1, x_{1}, \ldots, x_{m}$ and assume $\ell>m r$. Then one at least of the $\ell$ numbers

$$
x_{1} \lambda_{1 j}+\cdots+x_{m} \lambda_{m j} \quad(j=1, \ldots, \ell)
$$

is transcendental.

When $1, x_{1}, \ldots, x_{m}$ are linearly independent over $\overline{\mathbb{Q}}$, the condition $\ell>m r$ in Corollary 3.5 for getting a transcendental number is the same as in Theorem 2.11 for getting an element outside of $\widetilde{\mathcal{L}}$. However the 
former result holds with arbitrary complex numbers $x_{i}$ but restricts $\lambda_{i j}$ to be in $\mathcal{L}$, while the later one, namely Theorem 2.11, requires $\Lambda_{i} \in \widetilde{\mathcal{L}}$ and allows $\Lambda_{i j}$ in $\widetilde{\mathcal{L}}$.

\section{Elliptic Case}

The previous study involves the multiplicative group $\mathbf{G}_{m}$. Similar statements hold for an elliptic curve $\mathcal{E}$ which is defined over $\overline{\mathbb{Q}}$ : we replace the field $\mathbb{Q}$ by the field $\mathbb{k}_{\mathcal{E}}=$ End $\mathcal{E} \otimes_{\mathbb{Z}} \mathbb{Q}$ of endomorphisms of $\mathcal{E}$, the $\mathbb{Q}$-vector space $\mathcal{L}$ by the $\mathbb{k}_{\mathcal{E}}$-vector space $\mathcal{L}_{\mathcal{E}}$ of elliptic logarithms of algebraic points on $\mathcal{E}$, namely

$$
\mathcal{L}_{\mathcal{E}}=\exp _{\mathcal{E}}^{-1} E(\overline{\mathbb{Q}})
$$

and the $\overline{\mathbb{Q}}$-vector space $\widetilde{\mathcal{L}}$ by the $\overline{\mathbb{Q}}$-vector space $\widetilde{\mathcal{L}}_{\mathcal{E}}$ spanned by 1 and $\mathcal{L}_{\mathcal{E}}$ in $\mathbb{C}$.

Recall that the elliptic curve $\mathcal{E}$ has complex multiplication or is a $C M$ curve if $\mathbb{k}_{\mathcal{E}} \neq \mathbb{Q}$, in which case $\mathbb{k}_{\mathcal{E}}$ is an imaginary quadratic field.

The Weierstrass elliptic function $\wp$ associated to $\mathcal{E}$ satisfies a differential equation

$$
\wp^{\prime 2}=4 \wp^{3}-g_{2} \wp-g_{3}
$$

with algebraic invariants $g_{2}, g_{3}$; from the definition it follows that $\mathcal{L}_{\mathcal{E}}$ is the set of elliptic logarithm of algebraic points of $\wp$, namely the set of complex numbers $u$ such that either $u$ is a period of $\wp$ or else $\wp(u)$ is algebraic.

A special case of the elliptico-toric Conjecture of C. Bertolin in [1] is the following elliptic analog of Conjecture 1.1.

ConjeCture 4.1. (Algebraic Independence of Elliptic Logarithms of Algebraic Numbers). Let $u_{1}, \ldots, u_{n}$ be $\mathbb{k}_{\mathcal{E}}$-linearly independent elements of $\mathcal{L}_{\mathcal{E}}$. Then $u_{1}, \ldots, u_{n}$ are algebraically independent.

From this conjecture one readily deduces elliptic analogs to all the statements in $\S 1$ : one replaces the $\overline{\mathbb{Q}}$-vector space $\widetilde{\mathcal{L}}$ by the $\overline{\mathbb{Q}}$-vector space $\widetilde{\mathcal{L}}_{\mathcal{E}}$ consisting of linear combinations

$$
\beta_{0}+\beta_{1} u_{1}+\cdots+\beta_{n} u_{n}
$$

of elliptic logarithms $u_{i} \in \mathcal{L}_{\mathcal{E}}$ with algebraic coefficients $\beta_{j}$.

Elliptic analogs of the results in $\S 2$ deserve to be considered - we plan to do it elsewhere. Here we content ourselves with two transcendence statements (compare with Corollary 2.12) whose proofs are given in $\S 5$.

THEOREM 4.2. Let $\wp$ be a Weierstrass elliptic functions with algebraic invariants $g_{2}, g_{3}$. For $1 \leq j \leq 7$, let $\lambda_{j} \in \mathcal{L}$ and $u_{j} \in \mathcal{L}_{\mathcal{E}}$. Assume that the seven points $\left(\lambda_{1}, u_{1}\right), \ldots,\left(\lambda_{7}, u_{7}\right)$ are $\mathbb{Q}$-linearly independent in 
$\mathbb{C}^{2}$. Assume also $\lambda_{1} \neq 0$ and $u_{1} \neq 0$. Then one at least of the six numbers

$$
\lambda_{1} u_{j}-\lambda_{j} u_{1} \quad(j=2, \ldots, 7)
$$

is transcendental.

Furthermore, if $u_{1}$ is a period $\omega$ of $\wp$ and $\lambda_{1}=2 i \pi$, then one at least of the four numbers

$$
2 i \pi u_{j}-\lambda_{j} \omega \quad(j=2, \ldots, 5)
$$

is transcendental.

THEOREM 4.3. Let $\wp$ and $\wp^{*}$ be two Weierstrass elliptic functions with algebraic invariants $g_{2}, g_{3}$ and $g_{2}^{*}, g_{3}^{*}$ respectively. For $1 \leq j \leq 9$, let $u_{j}$ (resp. $u_{j}^{*}$ ) be an elliptic logarithm of an algebraic point of $\wp$ (resp. $\left.\wp^{*}\right)$. Assume that the nine points $\left(u_{1}, u_{1}^{*}\right), \ldots,\left(u_{9}, u_{9}^{*}\right)$ are $\mathbb{Q}$-linearly independent in $\mathbb{C}^{2}$. Assume further either that the elliptic curves $\mathcal{E}$ and $\mathcal{E}^{*}$ are non isogeneous and $u_{1} u_{1}^{*} \neq 0$, or else that $\mathcal{E}=\mathcal{E}^{*}$ and that the two numbers $u_{1}, u_{1}^{*}$ are linearly independent over $\mathbb{k}_{\mathcal{E}}$. Then one at least of the eight numbers

$$
u_{j} u_{1}^{*}-u_{j}^{*} u_{1} \quad(j=2, \ldots, 9)
$$

is transcendental.

Furthermore, if $u_{1}$ is a period $\omega$ of $\wp$ and $u_{1}^{*}$ a period $\omega^{*}$ of $\wp^{*}$, then one at least of the six numbers

$$
u_{j} \omega^{*}-u_{j}^{*} \omega \quad(j=2, \ldots, 7)
$$

is transcendental.

Remark. From the elliptico-toric Conjecture of C. Bertolin in $[\mathbf{1}]$ one deduces that, under the assumptions of Theorem 4.3, the number

$$
u_{2} u_{1}^{*}-u_{2}^{*} u_{1}
$$

is transcendental. More precisely, in the case where $\mathcal{E}$ and $\mathcal{E}^{*}$ are non isogeneous, her conjecture implies that the number $u_{1} u_{1}^{*}$ is transcendental, and that the transcendence degree $t$ over $\mathbb{Q}$ of the field $\mathbb{Q}\left(u_{1}, u_{2}, u_{1}^{*}, u_{2}^{*}\right)$ satisfies

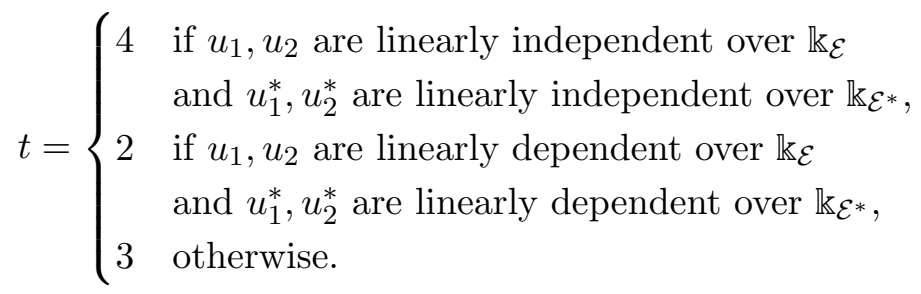


In particular if we denote by $\left(\omega_{1}, \omega_{2}\right)$ (resp. $\left.\left(\omega_{1}^{*}, \omega_{2}^{*}\right)\right)$ a pair of fundamental periods of $\wp$ (resp. of $\left.\wp^{*}\right)$, then, according to Bertolin's Conjecture, the number

$$
\omega_{2} \omega_{1}^{*}-\omega_{2}^{*} \omega_{1}
$$

is transcendental. I wish to thank H. Shiga who pointed out to me that such numbers occur as periods of $K 3$ surfaces (we refer to the appendix). His remark was the initial motivation for this paper.

\section{Proofs of Theorems 4.2 and 4.3}

The proofs of Theorems 4.2 and 4.3 rely on the following special case of the Algebraic Subgroup Theorem ([10] Th. 1.1 and [11] Th. 4.1)the full statement deals with a vector subspace $\mathcal{V}$, here we need only to consider a hyperplane.

TheOREM 5.1. Let $d_{0}, d_{1}, d_{2}$ be three non negative integers with $d=d_{0}+d_{1}+d_{2}>0$. Let $G_{2}$ be a commutative algebraic group over $\overline{\mathbb{Q}}$ of dimension $d_{2}$ and set $G=\mathbf{G}_{a}^{d_{0}} \times \mathbf{G}_{m}^{d_{1}} \times G_{2}$. Let $\mathcal{V}$ be a hyperplane of the tangent space $T_{e}(G)$ and $Y$ a finitely generated subgroup of $\mathcal{V}$ of rank $\ell$ such that $\exp _{G}(Y) \subset G(\overline{\mathbb{Q}})$. Let $\kappa$ be the $\mathbb{Z}$-rank of $\mathcal{V} \cap$ ker $\exp _{G}$. Assume

$$
\ell>(d-1)\left(d_{1}+2 d_{2}-\kappa\right) .
$$

Then $\mathcal{V}$ contains a non-zero algebraic Lie subalgebra of $T_{e}(G)$ defined over $\overline{\mathbb{Q}}$.

Proof of Theorem 4.2. Consider the matrix

$$
\left(\begin{array}{llll}
\lambda_{1} & \lambda_{2} & \ldots & \lambda_{\ell} \\
u_{1} & u_{2} & \ldots & u_{\ell}
\end{array}\right)
$$

where $\ell=7$ in general, unless $\lambda_{1}=2 i \pi$ and $u_{1}$ is a period $\omega$ of $\wp$, in which case $\ell=5$. Assume that the $\ell$ columns of $M$ are linearly independent over $\mathbb{Q}$ and that the $\ell$ numbers

$$
\gamma_{j}=u_{1} \lambda_{j}-\lambda_{1} u_{j} \quad(j=1, \ldots, \ell)
$$

are algebraic. Notice that $\gamma_{1}=0$.

Define $G=\mathbf{G}_{a} \times \mathbf{G}_{m} \times \mathcal{E}, d_{0}=1, d_{1}=1, d_{2}=1, G_{2}=\mathcal{E}, d=3$. Let $\mathcal{V}$ be the hyperplane

$$
z_{0}=u_{1} z_{1}-\lambda_{1} z_{2}
$$

in $\mathbb{C}^{3}$ and $Y=\mathbb{Z} y_{1}+\cdots+\mathbb{Z} y_{\ell}$ the subgroup of $\mathcal{V}$ of rank $\ell$ with

$$
y_{j}=\left(\gamma_{j}, \lambda_{j}, u_{j}\right), \quad(1 \leq j \leq \ell) .
$$


From the assumptions $\lambda_{1} \neq 0$ and $u_{1} \neq 0$ it follows that $\mathcal{V}$ does not contain any non-zero Lie subalgebra of $T_{e}(G)$. In case $\left(\lambda_{1}, u_{1}\right)=(2 i \pi, \omega) \in$ ker $\exp _{G}$, we have $\kappa \geq 1$. Since

$$
(d-1)\left(d_{1}+2 d_{2}-\kappa\right)= \begin{cases}6 & \text { if } \kappa=0 \\ 4 & \text { if } \kappa=1,\end{cases}
$$

Theorem 5.1 gives a contradiction.

Proof of Theorem 4.3. Let $\ell$ be a positive integer and $u_{1}, \ldots, u_{\ell}$ (resp. $u_{1}^{*}, \ldots, u_{\ell}^{*}$ ) be elliptic logarithms of algebraic points of $\wp$ (resp. $\left.\wp^{*}\right)$. Assume that $\left(u_{1}, u_{1}^{*}\right), \ldots,\left(u_{\ell}, u_{\ell}^{*}\right)$ are $\mathbb{Q}$-linearly independent in $\mathbb{C}^{2}$ and that the $\ell$ numbers

$$
\gamma_{j}=u_{j} u_{1}^{*}-u_{j}^{*} u_{1} \quad(j=1, \ldots, \ell)
$$

are algebraic.

Denote by $\mathcal{E}\left(\right.$ resp. $\left.\mathcal{E}^{*}\right)$ the elliptic curve associated to $\wp$ (resp. $\left.\wp^{*}\right)$. In Theorem 5.1 take $G=\mathbf{G}_{a} \times \mathcal{E} \times \mathcal{E}^{*}, d_{0}=1, d_{1}=0, d_{2}=2, d=3$. Let $\mathcal{V}$ be the hyperplane

$$
z_{0}=u_{1}^{*} z_{1}-u_{1} z_{2}
$$

of $\mathbb{C}^{3}$ and define $Y=\mathbb{Z} y_{1}+\cdots+\mathbb{Z} y_{\ell}$ with

$$
y_{j}=\left(\gamma_{j}, u_{j}, u_{j}^{*}\right), \quad(1 \leq j \leq \ell) .
$$

The assumption that either the elliptic curves $\mathcal{E}$ and $\mathcal{E}^{*}$ are non isogeneous and $u_{1} u_{1}^{*} \neq 0$, or else $\mathcal{E}=\mathcal{E}^{*}$ and $u_{1}, u_{1}^{*}$ are linearly independent over $\mathbb{k}_{\mathcal{E}}$, implies that $\mathcal{V}$ does not contain a non-zero Lie subalgebra of $T_{e}(G)$. Hence from Theorem 5.1 we deduce that the rank $\ell$ of $Y$ is bounded by

$$
\ell \leq(d-1)\left(d_{1}+2 d_{2}\right)=8 .
$$

Furthermore, if $u_{1}$ is a period of $\wp$ and $u_{1}^{*}$ a period of $\wp^{*}$, then the point $\left(0, u_{1}, u_{1}^{*}\right)$ belongs to $\mathcal{V} \cap \operatorname{ker} \exp _{G}$, hence $\kappa \geq 1$ and

$$
\ell \leq(d-1)\left(d_{1}+2 d_{2}-1\right)=6 .
$$

\section{References}

[1] C. Bertolin - Périodes de 1-motifs et transcendance, J. Number Theory 97 (2002), no. 2, p. 204-221.

[2] G. DiAZ - La conjecture des quatre exponentielles et les conjectures de D. Bertrand sur la fonction modulaire, J. Théor. Nombres Bordeaux 9 (1997), no. 1, p. 229-245. 
[3] Utilisation de la conjugaison complexe dans l'étude de la transcendance de valeurs de la fonction exponentielle, J. Théor. Nombres Bordeaux (2004), à paraître.

[4] N. I. FEL'DMAN \& Y. V. NESTERENKO - Transcendental numbers, in Number theory, IV, Encyclopaedia Math. Sci., vol. 44, Springer, Berlin, 1998, p. 1-345.

[5] S. LANG - Introduction to transcendental numbers, Addison-Wesley Publishing Co., Reading, Mass.-London-Don Mills, Ont., 1966.

[6] K. RamachandRA - Contributions to the theory of transcendental numbers. I, II, Acta Arith. 14 (1967/68), 65-72; ibid. (1967/1968), p. 73-88.

[7] D. Roy - Matrices whose coefficients are linear forms in logarithms, J. Number Theory 41 (1992), no. 1, p. 22-47.

[8] - Points whose coordinates are logarithms of algebraic numbers on algebraic varieties, Acta Math. 175 (1995), no. 1, p. 49-73.

[9] T. SchneIDER - Introduction aux nombres transcendants, Traduit de l'allemand par P. Eymard, Gauthier-Villars, Paris, 1959.

[10] M. WALDSCHMIDT - On the transcendence methods of Gel' fond and Schneider in several variables, in New advances in transcendence theory (Durham, 1986), Cambridge Univ. Press, Cambridge, 1988, p. 375-398.

[11] _ Dependence of logarithms of algebraic points, in Number theory, Vol. II (Budapest, 1987), Colloq. Math. Soc. János Bolyai, vol. 51, North-Holland, Amsterdam, 1990, p. 1013-1035.

[12] _ Diophantine approximation on linear algebraic groups, Grundlehren der Mathematischen Wissenschaften [Fundamental Principles of Mathematical Sciences], vol. 326, Springer-Verlag, Berlin, 2000.

Institut de Mathématiques de Jussieu - UMR 7586 Du CNRS, Université

P. et M. Curie (Paris VI), 175 rue du Chevaleret, F-75013 Paris

E-mail address: miw@math.jussieu.fr

$U R L$ : http://www.math.jussieu.fr/ miw 


\title{
Appendix: Periods on the Kummer surface
}

\author{
Hironori Shiga
}

\section{Periods of a Kummer surface}

1.1. Recalling the Kummer surfaces. Let $\omega_{1}, \ldots, \omega_{4}$ be points on $(z, w)$-space $\mathbf{C}^{2}$ those are independent over $\mathbf{R}$. Let $T$ be a complex torus defined by

$$
\mathbf{C}^{2} /\left(\mathbf{Z} \omega_{1}+\ldots+\mathbf{Z} \omega_{4}\right)
$$

We consider an involution of $\mathbf{C}^{2}$

$$
\iota:(z, w) \mapsto(-z,-w) .
$$

We obtain a complex 2 -dimensional variety $V=T / \iota$. It has 16 singularities corresponding to 16 fixed points of the involution (namely the half period points and zero). By the resolution of these singularities we get a Kummer surface $S=S\left(\omega_{1}, \ldots, \omega_{4}\right)$. All these surfaces are diffeomorphic each other. We may construct $S$ by the following alternative manner: Make first the blow up processes at 16 half period points on $T$, let us denote $\tilde{T}$ the resulting complex surface. The involution $\iota$ is still acting on $\tilde{T}$, so we have $S=\tilde{T} / \iota$. In this situation we denote by $\pi$ the canonical projection $\tilde{T} \rightarrow S$.

We have four 1-cycles $\gamma_{1}, \ldots, \gamma_{4}$ on $T$ those correspond to $\omega_{1}, \ldots, \omega_{4}$, respectively. But as easily checked $\pi\left(\gamma_{i}\right)$ is homotopic to zero, consequently $S$ is simply connected.

As for the 2nd homology group $H_{2}(S, \mathbf{Z})$, we have the following cycles:

1) the 2-cycles represented by 16 exceptional divisors obtained by the resolution procedure related above, we denote by $D$ the sub $\mathbf{Z}$-module generated by these divisors,

2) $\operatorname{six} 2$-cycles $\sigma_{i j}=\pi\left(\gamma_{i} \times \gamma_{j}\right)$.

2000 Mathematics Subject Classification. 14J28.

Key words and phrases. K3 surfaces, Kummer surfaces, periods. 
These 22 cycles are independent in the $\mathbf{Z}$-module $H_{2}(S, \mathbf{Z})$, and they give a basis over $\mathbf{Q}$, but not necessarily a basis over $\mathbf{Z}$. We can say $\left\{\sigma_{i j}\right\}$ is a $\mathbf{Z}$ basis of the quotient module $H_{2}(S, \mathbf{Z}) / D$. Any way we have rank $H_{2}(S, \mathbf{Z})=22$. It indicates $S$ is a K3 surface.

1.2. Their Periods. The holomorphic 2 -form $d z \wedge d w$ on $\mathbf{C}^{2}$ induces that of $S$. We denote it by $\varphi$, it is the unique holomorphic 2 -form on $S$ up to a constant factor. Because $\pi$ is a $2: 1$ map, we have the relation

$$
\int_{\sigma_{i j}} \varphi=\frac{1}{2} \int_{\gamma_{i} \times \gamma_{j}} d z \wedge d w .
$$

So the period on $S$ is essentially the same as the period of the complex torus $T$.

Let us consider the case when $T$ is a product of two elliptic curves $E=\mathbf{C} / \mathbf{Z} \omega_{1}+\mathbf{Z} \omega_{2}$ and $E^{*}=\mathbf{C} / \mathbf{Z} \omega_{1}^{*}+\mathbf{Z} \omega_{2}^{*}$. Let $\gamma_{1}, \gamma_{2}, \gamma_{1}^{*}, \gamma_{2}^{*}$ be the 1-cycles on $T=E \times E^{*}$ corresponding to $\omega_{1}, \omega_{2}, \omega_{1}^{*}, \omega_{2}^{*}$, respectively. By putting $C_{1}=\gamma_{1} \times \gamma_{2}^{*}$ and $C_{2}=\gamma_{2} \times \gamma_{1}^{*}$ on $T$, we have a 2-cycle $\sigma=\pi\left(C_{1}-C_{2}\right)$ on $S$. Then we obtain

$$
\int_{\sigma} \varphi=\frac{1}{2}\left(\omega_{1} \cdot \omega_{2}^{*}-\omega_{2} \cdot \omega_{1}^{*}\right) .
$$

Generally it was difficult to show the transcendency of the period of the Kummer surface. As we observed above, even in the case of product type the argument is reduced to the quadratic relation among the periods of elliptic curves. We can refer only one example of explicit transcendental periods of the Kummer surface obtained by classical arguments (see [1]).

Let us consider an one parameter family of algebraic surfaces (it is a family of some Kummer sufaces)

$$
\Sigma(\mu): x y z(x+y+z+1)+\mu^{4}=0, \quad \mu \in \mathbf{C} .
$$

We note that it is essentially the same as the family

$$
X^{4}+Y^{4}+Z^{4}+1+k X Y Z=0, \quad k \in \mathbf{C} .
$$

The holomorphic differential is given by

$$
\omega=\frac{d x \wedge d y}{f_{z}(x, y, z)},
$$

where $f$ stands for the left hand side of the defining equation of $\Sigma(\mu)$. One 2-cycle on $\Sigma(\mu)$, saying $K_{0}$, is given by the lifting (near to the origin) of a torus $\{|y|=1 / 4\} \times\{|z|=1 / 4\}$ via the natural projection from $\Sigma(\mu)$ to the $(x, y)$ - space. Suppose $\mu$ is an algebraic number, then the period

$$
\int_{K_{0}} \omega
$$


is always transcendental. To prove it we can reduce the argument to the transcendency of the period of a hypergeometric curve.

\section{References}

[1] Narumiya, N and H. Shiga, The Mirror Map for a Family of K3 Surfaces Induced From the Simplest 3-Dimensional Reflexive Polytope, CRM Proc. Lecture Notes, 30 (2001), 139-161. MR 2002m:14030

Department of Mathematics, Faculty of Science, Chiba university, 133 YAYOI-CHO, INAGE-KU, CHIBA, 263-8522 JAPAN

E-mail address: shiga@math.s.chiba-u.ac.jp 